\title{
AN ANALYSIS OF FATIGUE CRACK INITIATION USING 2D ORIENTATION MAPPING AND FULL-FIELD SIMULATION OF ELASTIC STRESS RESPONSE
}

\author{
Clayton Stein ${ }^{1}$, Sukbin Lee ${ }^{1}$, Anthony Rollett ${ }^{1}$ \\ ${ }^{1}$ Carnegie Mellon University; 5000 Forbes Avenue; Pittsburgh, PA 15213, USA \\ email: claystein@cmu.edu
}

Keywords: fatigue, crack initiation, LSHR, crystallographic orientation

\begin{abstract}
A preliminary investigation into the microstructural factors potentially associated with fatigue crack initiation along coherent twin boundaries in the nickel-based superalloy LSHR is presented. Cracks appear to initiate predominantly along twin boundaries. Electron Back Scatter Diffraction (EBSD) maps, coupled with calculations of the local elastic stress point to an influence of the Schmid factor. The length of the twin appears, however, to be more strongly correlated with the tendency to crack.
\end{abstract}

\section{Introduction}

Nickel-base superalloys are important engineering materials, particularly in the demanding application in various parts of gas turbines for aerospace and, increasingly, the ground-based energy production which will become more important as more electrical energy productions shifts from fossil-based to less-steady renewable sources. The continuing performance and efficiency increases demanded by these applications require operation at higher temperatures and expectations of longer fatigue lifetimes. Fatigue failures depend not only on crack growth rates but also crack initiation, therefore predicting and extending fatigue life requires not only the implementation of crack growth models from fracture mechanics, but also a better understanding of the relationship between microstructure and the initiation of small fatigue cracks at the length scale of individual grains $[1-3]$.

\section{Approach}

The overall approach of the present work is to characterize fatigued nickel-base superalloy specimens, identify fatigue microcracks in the surface, and combine orientation data with a simulation of the stress response to investigate any trends in the microstructures. Initial work is limited to two-dimensional automated electron backscatter diffraction (EBSD) mapping of microcracks, and simulation of the material stress state using the corresponding $2 \mathrm{~d}$ map of grain orientation information. The simulation is achieved via a fast Fourier transform (FFT) technique $[4,5]$ providing a full-field model of the elastic stress response.

The specimens are $3 \mathrm{~mm}$ wide, flat dog bone samples of the advanced disk alloy LSHR [6]. The nominal composition of LSHR in weight percent is $3.5 \mathrm{Al}, 0.03$ $\mathrm{B}, 0.03 \mathrm{C}, 20.7 \mathrm{Co}, 12.5 \mathrm{Cr}, 2.7 \mathrm{Mo}, 1.5 \mathrm{Nb}, 1.6 \mathrm{Ta}, 3.5 \mathrm{Ti}$, $4.3 \mathrm{~W}, 0.05 \mathrm{Zr}$, bal $\mathrm{Ni}$. The processing consisted of atomization in argon, atomized powder being passed through screens of -270 mesh to give powder particle diameters of no more than about $55 \mu \mathrm{m}$. The powder is then sealed in a stainless steel container, hot compacted, and extruded at a reduction ratio of 6:1. Rods of 0.5 inch diameter and about 6 inch length were induction heated in the middle region above the gamma prime solvus temperature to produce a supersolvus, coarse grain structure. Thin (approximately $1 \mathrm{~mm}$ thickness) specimens were then EDM-machined from these rods. Average grain size in the coarse-grained and fine-grained regions were about 23 and $4 \mu \mathrm{m}$ respectively. A secondary electron image of the microstructure showing gamma prime, as well as an example of a surface microcrack, is depicted in Fig. 1. Figure 2 depicts a grain size distribution. This distribution was calculated by fitting ellipses to a map of the microstructure, and plotting the results based on the length of the major axes. Note that the fitted line in this and later cumulative probability plots represents an exactly normal distribution, given as comparison to the experimental distribution. The test specimens received an electropolish and were fatigued in tension-tension under conditions sufficient to generate surface cracks (test parameters are give in Table I). Among potential crack initiation sites, as indicated by sample replications, four surface cracks were selected for characterization and analysis. One of the test specimens was sectioned in support of future efforts to characterize and analyze the three-dimensional microstructure. The large-area EBSD mapping of these 


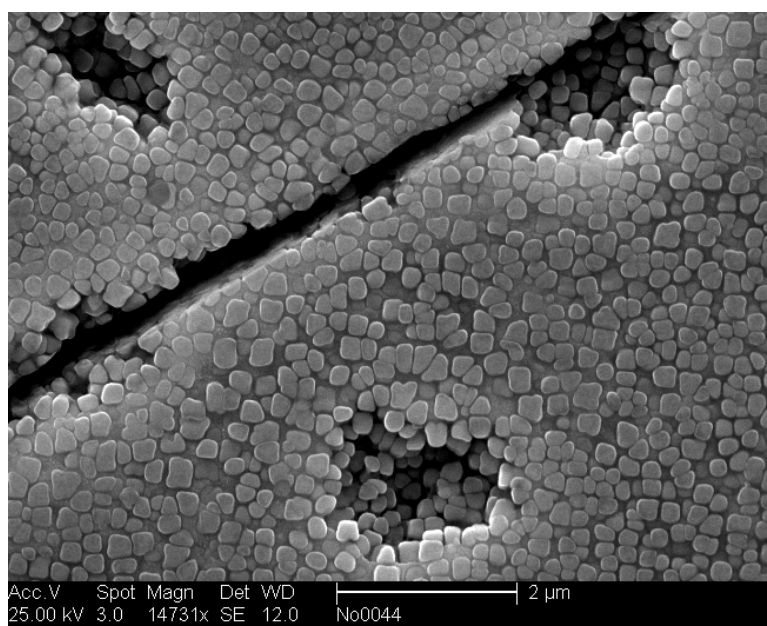

Figure 1: Secondary electron image of a microcrack on the surface of an electropolished fatigue test specimen.

Table I: Test Parameters

\begin{tabular}{|l|l|}
\hline maximum stress & $1050 \mathrm{MPa}$ \\
\hline stress ratio, $\mathrm{R}$ & 0.05 \\
\hline frequency & $10 \mathrm{~Hz}$ \\
\hline temperature & $23^{\circ} \mathrm{C}$ \\
\hline number of cycles, $\mathrm{N}$ & 37,500 \\
\hline
\end{tabular}

surface cracks provided the orientation information necessary for both the boundary analysis, a description of which follows, and as input for the elastic FFT simulation. For comparison, a simple Schmid factor calculation, assuming a constant stress state everywhere, is also presented. The spectral method is an alternative to finite element modeling, used in this instance because of its convenience and speed. The FFT-based elastic simulation code was developed by R. Lebensohn [5], based upon a simulation model developed by Moulinec and Suquet [4] for the analysis of composite structures. This simulation approach was adopted in the present study due to a number of important features. First, most characterization techniques yield microstructural data on a discretized grid. The FFT-based approach takes an orientation map as direct input, precluding the need for a mesh generation procedure as with finite element-based simulations. In addition, improvements in characterization techniques, specifically in $3 \mathrm{D}$ characterization, have led to increasingly large datasets requiring more efficient simulation techniques. The FFT-based approach is much less computationally intensive, with results which

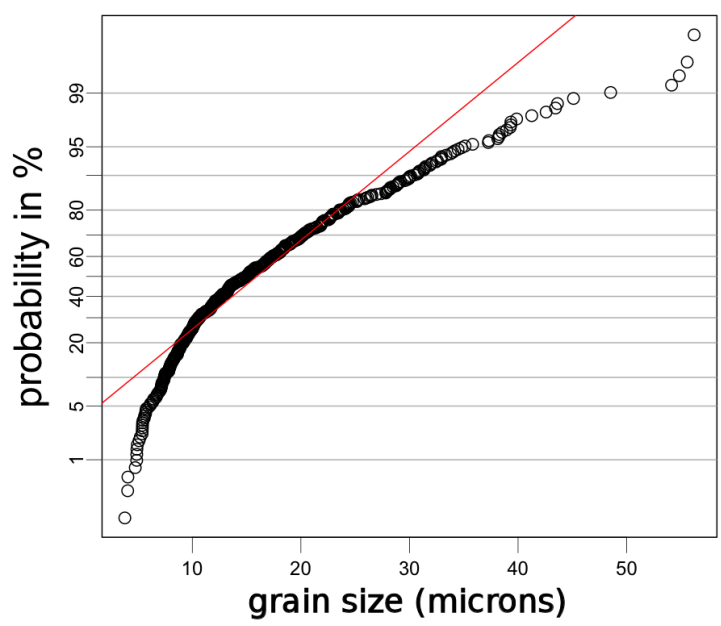

Figure 2: A typical distribution of grain sizes, measured by fitting ellipses to the grains and taking the length of the major ellipse axis. The fitted line in this cumulative probability plots represents an exactly normal distribution, given as comparison to the experimental distribution.

have been shown to compare favorably to finite element simulations [7].

The simulation of elastic stress response imposes a strain boundary condition, and the reported stress values will of course depend on the amount of strain. As it is a purely elastic calculation with no regard for the yield stress, the relative values of stress on different systems, as affected by the elastic anisotropy, are of importance rather than the absolute values.

The boundary analysis consists of grain segmentation using the commercial TSL EBSD analysis software, followed by segmentation and smoothing of boundary segments using in-house code which sorts boundaries by type according to their coincident site lattice (CSL) $\Sigma$ designation. The $\Sigma 3$ boundaries were further sorted into a list of likely coherent boundaries by calculation of the surface trace normal. Schmid factors were calculated, for those grains that are adjacent to coherent $\Sigma 3$ boundaries, not by taking the maximum value from amongst all available face centered cubic (fcc) slip systems (as for example in Fig. 3, but by selection of the maximum value from only the boundary-parallel slip planes. An example is depicted in Fig. 4, with the maximum crack-neighboring 
point-by-point maximum Schmid factors

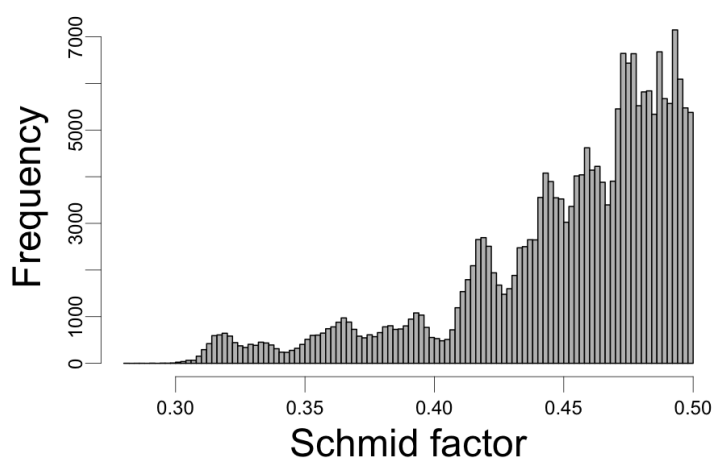

Figure 3: A typical distribution of maximum Schmid factor values, taking the maximum from all 12 possible slip systems.

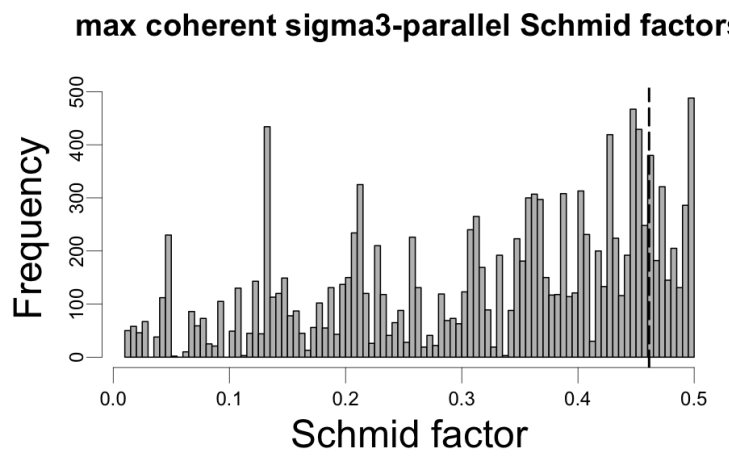

Figure 4: A distribution of maximum Schmid factors from amongst coherent $\Sigma 3$ boundary-parallel slip planes, with the maximum crack-neighboring value highlighted by the dashed line.

value highlighted by the dashed line. These plots are shown for comparison to the more refined calculation of maximum resolved shear stress along coherent $\Sigma 3$ boundary parallel planes, calculated by combining the segmented boundary list with the corresponding simulated stress field data.

\section{Results and Analysis}

The observed cracks occur along likely coherent $\Sigma 3$ boundaries, oriented favorably for slip along boundary parallel slip directions. Figure 5 depicts one of the cracks, and the corresponding EBSD map appears in the following figure (Fig. 6), with the horizontal direction corresponding to the specimen tensile axis.

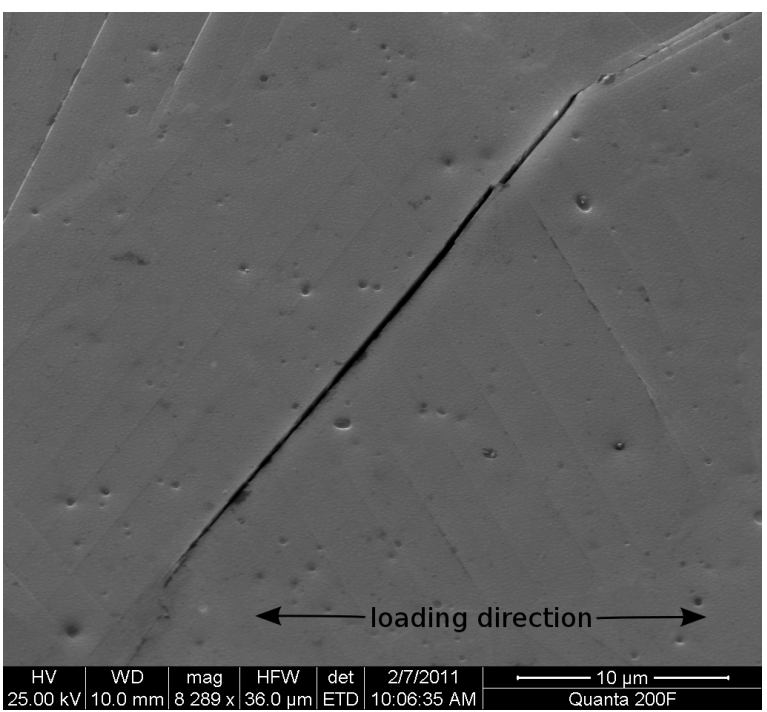

Figure 5: Secondary electron image of a microcrack on the surface of an electropolished fatigue test specimen. The crack occurs along a coherent $\Sigma 3$ boundary.

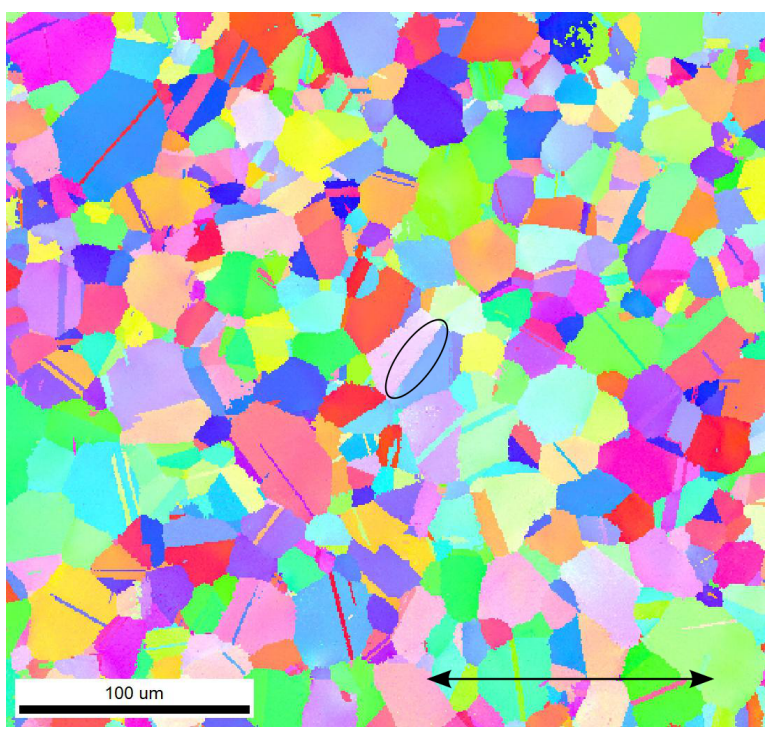

Figure 6: EBSD map of the region surrounding the crack depicted in the previous figure. The arrow indicates the loading direction. The crack boundary is circled near the center of the image.

The segmentation by boundary type is given in Fig. 7, with $\Sigma 3$ boundaries in yellow, coherent $\Sigma 3$ boundaries in red, and general boundaries in blue (note: the PDF 


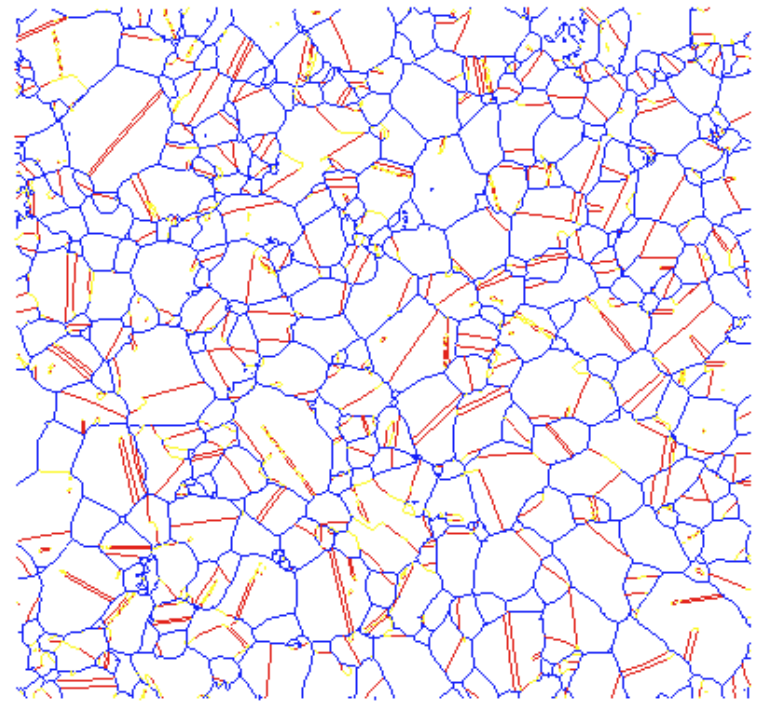

Figure 7: A segmentation map of the region depicted in Figure 6, colored by boundary type as described in the text.

file on the accompanying $\mathrm{CD}$ should be examined to see the colors). In order to visualize the distribution of resolved shear stresses (RSS), the coherent boundaries are colored according to the maximum calculated value from either side of the boundary in Fig. 8. Other researchers have described a combination of microstructural features associated with surface crack initiation in similar alloys under similar test conditions [8-11], namely the presence of $\Sigma 3$ twins, favorably oriented for slip, with some indication that grain size also plays a role. Initial results presented here support this same combination of microstructural features. Cracks were observed along candidate coherent $\Sigma 3$ boundaries. However, such boundaries make up approximately $30 \%$ by length of all surfaces characterized. Microcracks also occurred along those boundaries favorably oriented for slip, at least to a first order, as indicated by a constant stress state assumption (i.e. Schmid factors). Here again, this appears to be a necessary but not sufficient condition for crack initiation. Although a full-field simulation of the elastic stress response does indicate relatively high RSS values for crack-adjacent grains in a few instances, there is a broad spread of RSS values even for this small sampling of cracks. In terms of boundary length, crack-initiating boundaries do fall in the upper tail of the distribution.

Initiation of LCF cracks in these specimens is

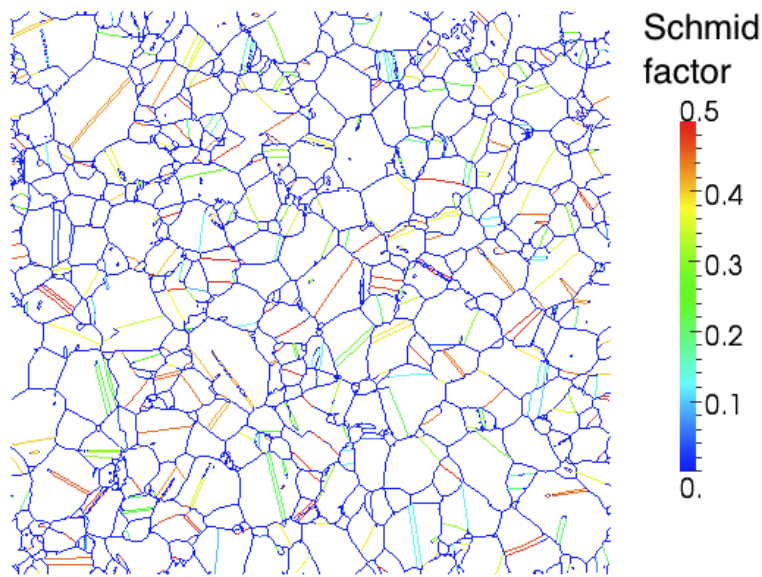

Figure 8: The same region is depicted with $\Sigma 3$ boundaries colored according to the value of the maximum Schmid factor chosen from amongst $\Sigma 3$ boundaryparallel slip planes.

likely to be due to a combination of factors, with long, favorably-oriented twin boundaries favored, but more work is needed to develop a mechanism for crack initiation, establish greater statistical certainty and test models that are consistent with the thus-far observed trend. Despite their ostensibly low grain boundary energies, these coherent boundaries might offer a greater opportunity for slip due to their plane orientation relative to the loading axis, and the longest of these boundaries may be susceptible to a greater accumulation of dislocation pileups and resulting stress concentrations. Although this might account for the unexpected initiation of cracks along such boundaries, a more rigorous statistical analysis of the relative importance of RSS versus boundary length is required.

Cumulative probability plots summarizing the results for the four crack regions characterized are depicted in the following figures. Plots of maximum RSS values, from grains adjacent to coherent $\Sigma 3$ boundaries, are depicted in Fig. 9, with a cumulative probability plot of RSS values for all regions combined given in Fig. 10. Crack-adjacent values are indicated by vertical lines in each figure. The boundary length distribution of $\Sigma 3$ boundaries is depicted in Fig. 11, in which data from all four crack regions are combined.

\section{Summary}

After observation of fatigue crack initiation along $\Sigma 3$ twin boundaries, and simulation of the tensile loading 

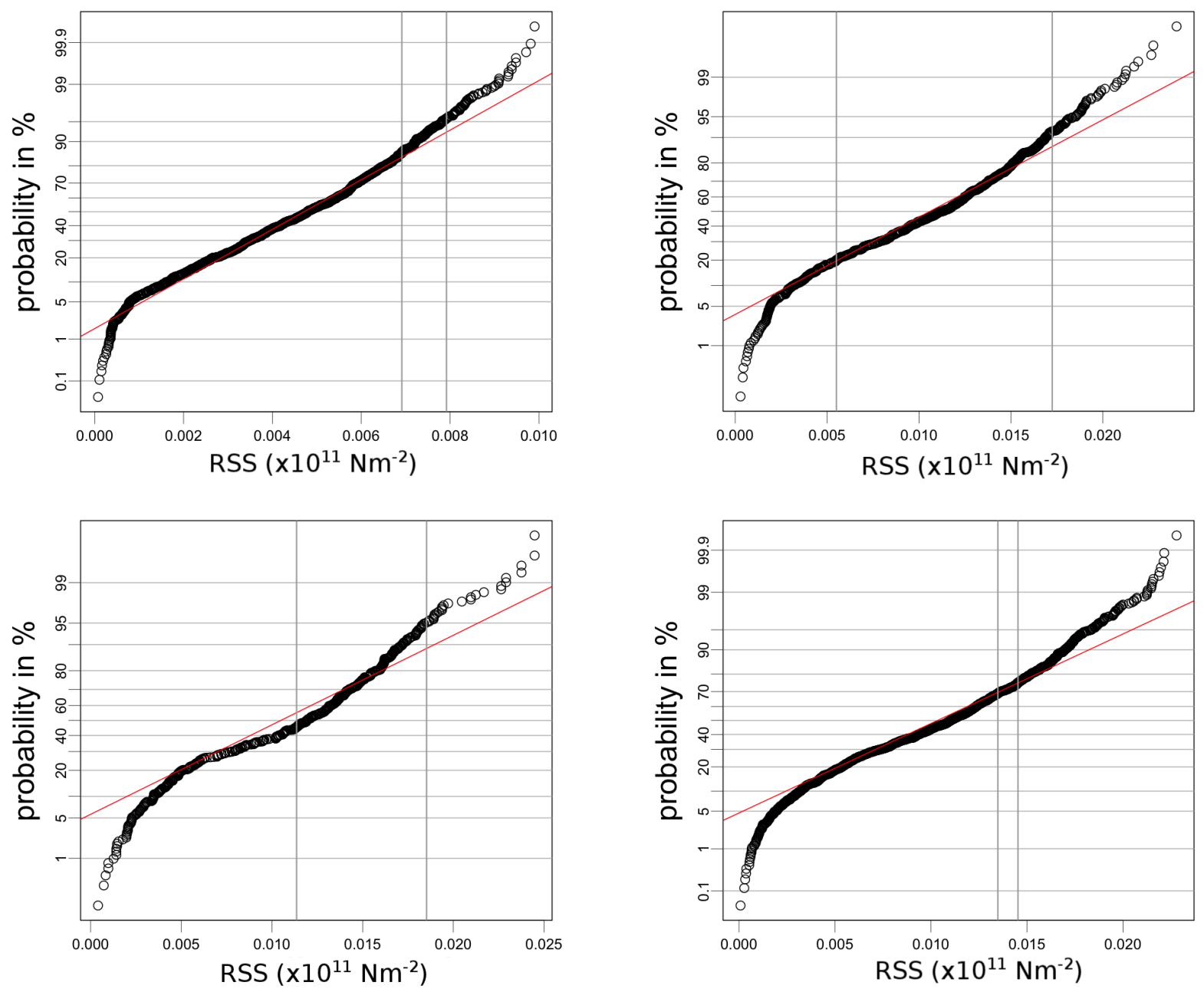

Figure 9: Cumulative probability plots of maximum resolved shear stress in $\Sigma 3$ boundary-adjacent grains, for each of four different microcrack regions. Vertical lines indicate values for the crack-adjacent grains. The fitted lines in these cumulative probability plots represent an exactly normal distribution, given as comparison to the experimental distribution.

elastic response of fatigued nickel-base superalloy specimens, the resolved shear stress along such boundaries has been estimated. The initial results point to the potential importance of boundary length for those coherent twin boundaries favorably oriented for slip activity. Aside from obtaining additional evidence to support this analysis, future work will employ full-field elastoviscoplastic simulation of the stress response under fatigue test conditions, as well as simulations based on orientation data from a three-dimensional volume. Not only will the three-dimensional microstructure improve the stress field simulation, it will provide additional microstructural metrics such as grain boundary area, and allow for the testing of more refined models for the initiation of microcracks in these materials.

\section{Acknowledgments}

This project is supported by an AFOSR Discovery Challenge Thrust grant \#FA9550-10-1-0213. The authors are grateful for the provision of materials and testing by the Air Force Research Laboratory at the WrightPatterson Air Force Base in Dayton, Ohio, courtesy of Drs. R. John and S. Jha. 


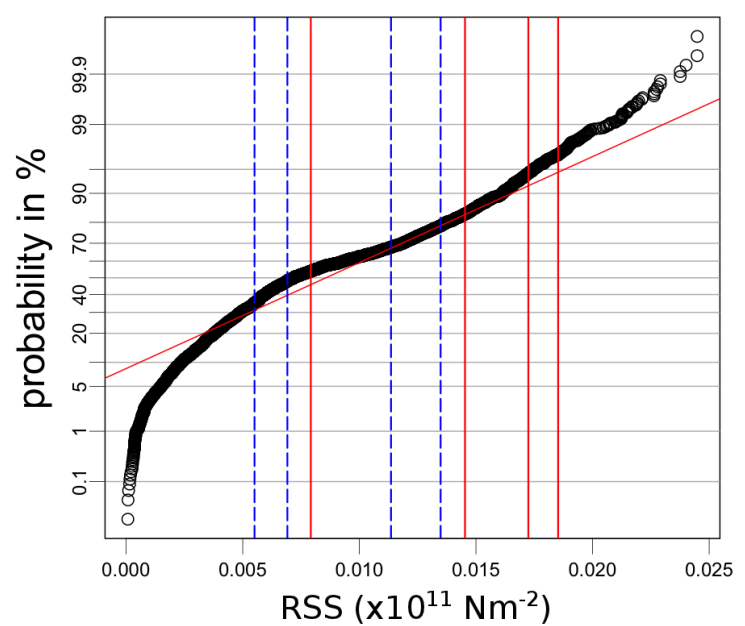

Figure 10: Cumulative probability plot of the combined EBSD data from the previous figure. Vertical lines indicate values for the crack-adjacent grains, with solid red lines corresponding to the larger, dashed blue lines the smaller, of the values from either side of a given crack. The fitted line represents an exactly normal distribution, given as comparison to the experimental distribution.

\section{References}

1. J.R. Brockenbrough, A.J. Hinkle, P.E. Magnusen, and R.J. Bucci, "Microstructurally based model of fatigue and growth" (NASA Conference Publication $3274,1994), 71-84$.

2. J.C. Newman Jr, "Advances in Fatigue and Fracture Mechanics Analyses for Aircraft Structures" (NASA/TM-2000-210084, 2000).

3. Subra Suresh, Fatigue of Materials 2nd ed. (Cambridge, UK: Cambridge University Press, 1998).

4. H. Moulinec and P. Suquet, "A numerical method for computing the overall response of nonlinear composites with complex microstructure," Computer Methods in Applied Mechanics and Engineering, 157 (1-2) (1998), 69-94.

5. R.A. Lebensohn, "N-site modeling of a 3D viscoplastic polycrystal using Fast Fourier Transform," Acta Materialia, 49 (14) (2001), 2723-2737.

6. T.P. Gabb, J. Gayda, J. Telesman, and P.T Kantzos, "Thermal and Mechanical Property Char-

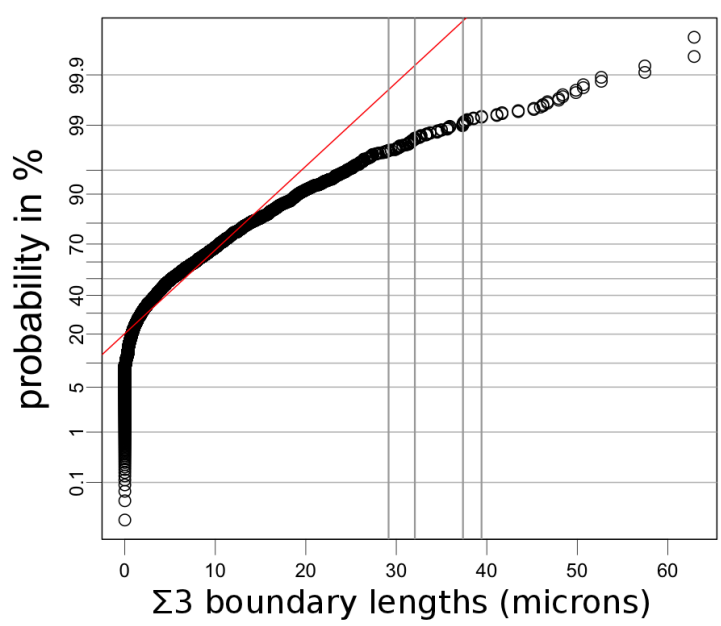

Figure 11: Cumulative probability distribution of coherent $\Sigma 3$ boundary lengths, for the combined EBSD data of four surface crack regions, with crack boundaries indicated by vertical lines. The fitted line represents an exactly normal distribution, given as comparison to the experimental distribution.

acterization of the Advanced Disk Alloy LSHR" (NASA/TM-2005-213645, 2005).

7. A. Prakash and R.A. Lebensohn, "Simulation of micromechanical behavior of polycrystals: finite elements versus fast Fourier transforms," Modelling and Simulation in Materials Science and Engineering, 17 (6) (2009), 064010.

8. J.S. Miao, T.M. Pollock, and J.W. Jones, "Crystallographic fatigue crack initiation in nickel-based superalloy René 88DT at elevated temperature," Acta Materialia, 57 (2009), 5964-5974.

9. A.W. Thompson and W.A. Backofen, "The effect of grain size on fatigue," Acta Metallurgica, 19 (7) (1971), 597-606.

10. A.W. Thompson, "The influence of grain and twin boundaries in fatigue cracking," Acta Metallurgica, 20 (9) (1972), 1085-1094.

11. A.W. Thompson and R.J. Bucci, "The dependence of fatigue crack growth rate on grain size," Metallurgical and Materials Transactions B, 4 (4) (1973), 1173-1175. 